\title{
A program for the analysis of intragroup interaction based on spatial dynamics
}

\author{
W. P. DODD \\ Computer Centre \\ and \\ E. JONES \\ Subdepartment of Ethology \\ University of Birmingham, Birmingham, England
}

\begin{abstract}
This paper describes the salient features of a computer program used in the analysis of the activity of a group of long-tail macaques. The principal feature concerns the time variation of the separation of selected dyads, although individual activity is also summarized. The program has been written such that it is adaptable to any enclosed group of Ss.
\end{abstract}

\section{INTRODUCTION}

As part of a continuing investigation into the social interaction of an enclosed group of long-tail macaques, the location of each macaque has been recorded at 2-min intervals over an $8 . \mathrm{h}$ period for a number of weeks. From these data, information has been derived regarding the relative separation of the Ss, their preferred locations, and some measure of their activity. Although originally there were only six Ss occupying an environment having 25 distinct locations, the data amassed are of sufficient quantity and the necessary calculations sufficiently repetitious for analysis of the data by computer to be almost an automatic choice.

In this paper, we describe the salient features of the computer program in the belief that it may be of use to others studying the behavior of any group of Ss within a confined environment. Apart from a few exceptions to be described later, the program has been written entirely in I.S.O. FORTRAN.

\section{DESCRIPTION OF AVAILABLE FACILITIES}

The aspect of the data with which we were most concerned was the interindividual distances. We wished to observe how these distances varied between different animals and how, for any selected dyad, the separation varied temporally throughout the 8 -h observation session and from one session to another. In particular, we were concerned with the mother-infant dyad and the spacial relationship of all individuals to the dominant male. A study could then be made of the variation of the latter two distances with the age of the Ss, since the group consisted of adults, juveniles, and infants, and the observation period extended over 18 months, during which further infants were born.

This increase in the size of the group emphasizes one of the necessary requirements of the program: that it must be easily adapted to accommodate varying numbers of Ss, and by logical extension, to varying numbers of locations within the environment, and to differing periods and intervals of observation. At one stage, we had planned to use a single array in which to store all the data on a dynamic allocation basis. However, we decided that such a structure would prove restrictive to other possible users who would probably wish to modify the program to include their own particular requirements. Instead, a number of storage arrays are used, and these are grouped according to which parameter of the quantity of data determines their size. This topic will be taken up later in the section on using the program.

It was our opinion that another essential requirement of the program was that a large part of the information produced should be represented in some pictorial fashion. Some of the information that we required could be produced on the line printer in the form of histograms, and we could use this output medium to summarize other information.

However, for the interindividual distances and their time variation, we chose to draw the graphs using a digital plotter. As these devices are comparatively slow, the program was supplied with a number of parameters to control, for example, whether to plot any graphs at all, for which dyads (pairs of Ss) the graphs were required, and the physical dimensions of the plots. We could then use the program initially with the plotting "switched off" to check the data for any punching errors, and only when the errors had been removed would the program be used to draw the interindividual distance plots for selected pairs of Ss.

We are more interested in the relative distances between pairs having a common member than in the absolute distances, and we were not, initially, interested in all pairs. So, for the purposes of these plots, we decided to reclassify the Ss into groups centered on a common (focal) member. We would then produce plots for the pairs consisting of the focal member and each of 
the other (dependent) members. The constitution and number of these groups would be supplied to the program as a run-time parameter and, so far as legibility permitted, the plots for a given group would be superimposed.

The interindividual distances were the principal information that we wished to extract from the data; however, the program provides further useful information. First, regarding the individual behavior of the Ss, the program can produce histograms of the location occupied by the $S$. These give the favored positions for each $S$ and for the group as a whole. They also give some indication of the activity of each $\mathrm{S}$. However, the histograms do not distinguish between a slow migration across the environment and a rapid motion back and forth. To obtain a more meaningful indication of the activity, the program sums the distances between consecutive locations occupied by the $S$. It is probable that the activity so measured is subject to a diumal variation which would be obscured by simply recording the histograms and distance traveled over the entire observation period. To overcome this limitation, the observation period can be divided into intervals and the histograms and distances recorded separately for each interval. Again, the length of the interval is a run-time parameter.

In addition to the histograms produced for each interval, the program produces summary histograms, and total "distance traveled," at the end of the observation period. A further table is constructed from the summary histograms in which the location occupation count is converted to a percentage, in case there are discrepancies in the number of observations or the interval between observations, from one observation period to another.

At the end of the observation period, further summary information is listed. The program produces a table of mean distances between all $S$ pairs and a count of the number of times that each pair were in contact. However, we are more interested in the relative separations of the various $S$ pairs than in the absolute distances, and so the program has been arranged to produce tables indicating these relative separations. Taking each $S$ in turn as a focus, a count is made for each of the other Ss of the number of times that $S$ is closest to the focal $S$, how many times it was second closest, and so on. These counts are then converted to percentages before being listed.

\section{FORM OF THE INPUT DATA}

The data required by the program can be conveniently divided into two sections, the fixed data and the observation data. The fixed data contain such things as the number and names of the Ss, of the locations of the Ss' environment, and the interlocation distances. It also contains the run-time parameters such as the interobservation time interval, the time interval after which we wish to print the location occupation histograms, and the parameters and group structure for

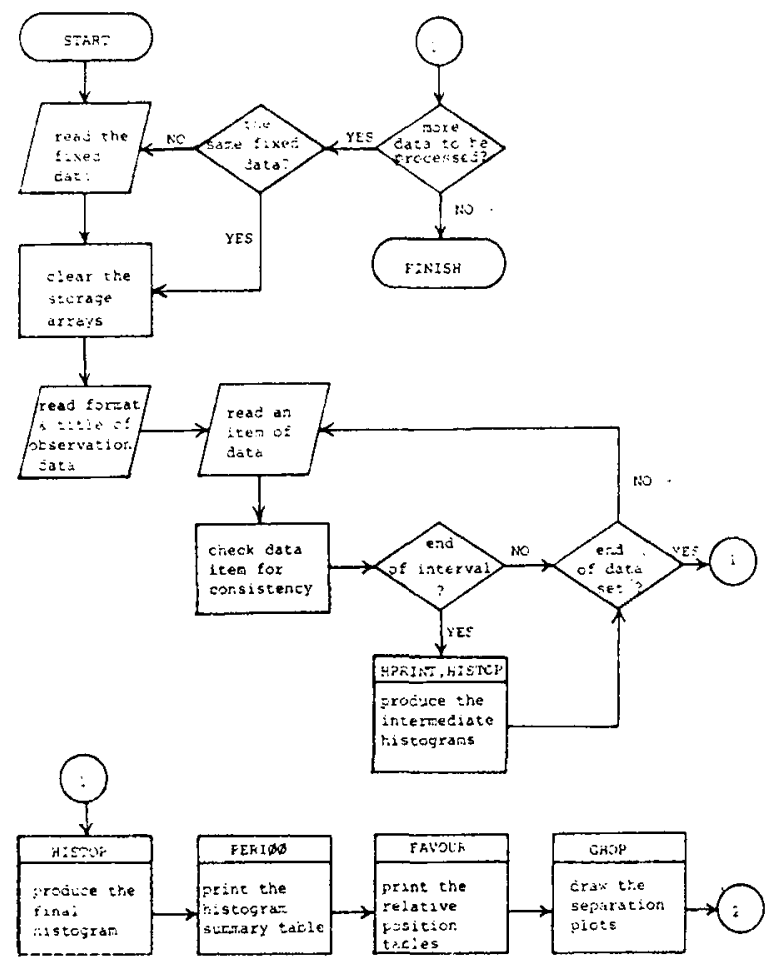

Fig. 1.

the inter-S distance plots. Full details conceming the fixed data are included in the program listing; however, we would like to make one comment here regarding the formats used to read in this data. Many of the quantities in the fixed data will be integers or floating point numbers, and these can be assigned a definite format. However, the names of the Ss and of the locations are read in as alphanumeric information, and owing to the differences in word size between computers we decided to read in the format for these quantities at run time. This permits the user a greater degree of control over the layout of the data cards, and does not impose any restrictions on the naming of either Ss or locations, subject only to the limit of fitting into the storage space allocated by the computer to a floating-point number (two words on the I.C.L. 1900 series, equivalent to eight letters).

The observation data section contains all the data pertinent to one observation period, together with a title associated with the data, such as the date of the observations, and a user-supplied format with which the observation data may be read. The individual observation data consist of the observation time and the location of each of the Ss, the format being usually arranged so that each observation of the colony would fit on to one card, unless of course the colony was large.

Many sets of observations may be supplied to the program for a single run, and they may or may not use the same set of fixed data. To distinguish the various observation sets, each one is terminated by a card containing a negative number in the location allocated 
by the format to the observation time. After reading this termination card and producing the relevant output, the program normally would attempt to read a new set of fixed data and would commence by reading the number of Ss. This number can be used as a flag to indicate whether to read a new set of fixed data, whether to use the old set, or whether to terminate the run, and it is assigned a positive, nega tive, or zero value accordingly. It is not possible to alter selectively a particular item of the fixed data; if even only one item is to be altered, the entire fixed data section must be supplied.

For convenience when processing many sets of information, the fixed data and the observation data are read on different input channels. Since the fixed data does not usually change for each set of observation data, it can be stored in the system file store, if available, and only the observation data and any necessary system commands need be supplied for each run.

\section{STRUCTURE OF THE PROGRAM}

The program consists of one principal routine, CHIMP, and a number of service routines, and as such the gross structure of the program is equivalent to that of CHIMP (q.v.). Figure 1 shows the flow diagram indicating both the procedures and the routines in which they are obeyed. All the service routines are connected with the various information output facilities provided by the program. The input of the fixed data and the input and processing of the observation data are all carried out in CHIMP, which then calls on the other routines when the end of a data set has been detected.

\section{DESCRIPTION OF THE ROUTINES}

\section{(1) Subroutine BOX (XLOW, XHIGH, YLOW, YHIGH)}

Called from: GHOP. Calls: POINT, JOIN.

This is a service routine, possibly included in some graphical output packages, for drawing a rectangle specified by the four arguments.

\section{(2) Subroutine CHIMP}

Called from: Main program. Calls: COMP8, FAVOUR, GHOP, HISTOP, HPRINT, PER $1 \emptyset \emptyset$.

This routine, which is effectively the main program, is in three sections. The first section reads, checks, and lists the fixed data, and it is here that the storage arrays are cleared. The second section processes the run data, having initially read in the data title and the format of the data cards. As each card is read in, it is checked for location name errors (usually mispunched cards) and for duplicated observations. For subsequent faster processing, the alphanumeric location names are here converted to integers, and if the data card is error free, the data are added into the location occupation array IHIST. This section of the routine also keeps the running sum of the distance traveled by each $S$ and of all the inter-S distances. Every data card must contain the time of the observation, and if this time corresponds to the end of the histogram output interval (see Section II), a call is made to HPRINT to initiate the printing. The final section of the routine is reached when all the data for an observation period has been read or when the observation storage arrays have been filled. This section contains a call to HISTOP to print the final histograms, to PER $1 \emptyset \emptyset$ for the summary table, to FAVOUR for the relative position tables, and to GHOP for the relative distance plots. In addition, CHIMP produces tables of the distance traveled by each $\mathrm{S}$ and of the mean distances between all pairs of Ss. The first section is then reentered in case further data require processing.

\section{(3) Subroutine COMMENTS}

This consists entirely of comment statements which describe more fully the format of the input data, and the specification of the required library routines. It also contains a description of all the common and local variables, and a flow chart of the program.

\section{(4) Subroutine FAVOUR}

Called from: CHIMP.

It is in this routine that the tables of the relative separations of all pairs of Ss are produced. To define the relative separation of a pair, we select one member of the pair to be the principal member, and then count how many other Ss are closer to the principal member than is the other member of the pair. This count plus one is the relative separation index (RSI). It is seen from this definition that the RSI is noncommutative, and that if there are N Ss in the group the RSI can only take on the values 1 to $\mathrm{N}-1$. Further, if there are two, or more, Ss at exactly the same distance from the principal member, a common situation in our study, then by the above definition the RSIs for the group would be, for example, 1, 2, 3, 3, 5. Subroutine FAVOUR carries out these calculations using four nested loops. The outer two loops define the principal and the other member of the pair, while the next loop is over the number of observations. For each observation, the distance between the pair is calculated and then in the innermost loop this distance is compared with the distances between the principal member and all the other Ss in the group. This comparison yields the RSI of the pair for each observation, and a count is made of the frequency with which each possible value occurs. Finally, these counts are converted to percentages before being printed.

\section{(5) Subroutine GHOP}

Called from: CHIMP. Calls: COPY8, Graphical Output Package routines.

In adapting the program for use on any other system, it is this routine that will possibly require modification, for it is here that the drawing of the relative distance plots is controlled. The specification of the graphical output routines is included with the program listing and is referred to briefly in Section VI. The first section of GHOP handles the definition of the plot area, and writes out the title associated with the data. The main section 
DATA FROM 14 EECEMBER 1972 RLN :2

DISTANCES RELATIVE TO PERCY

ARLO ALF BEBE ANN BETTY

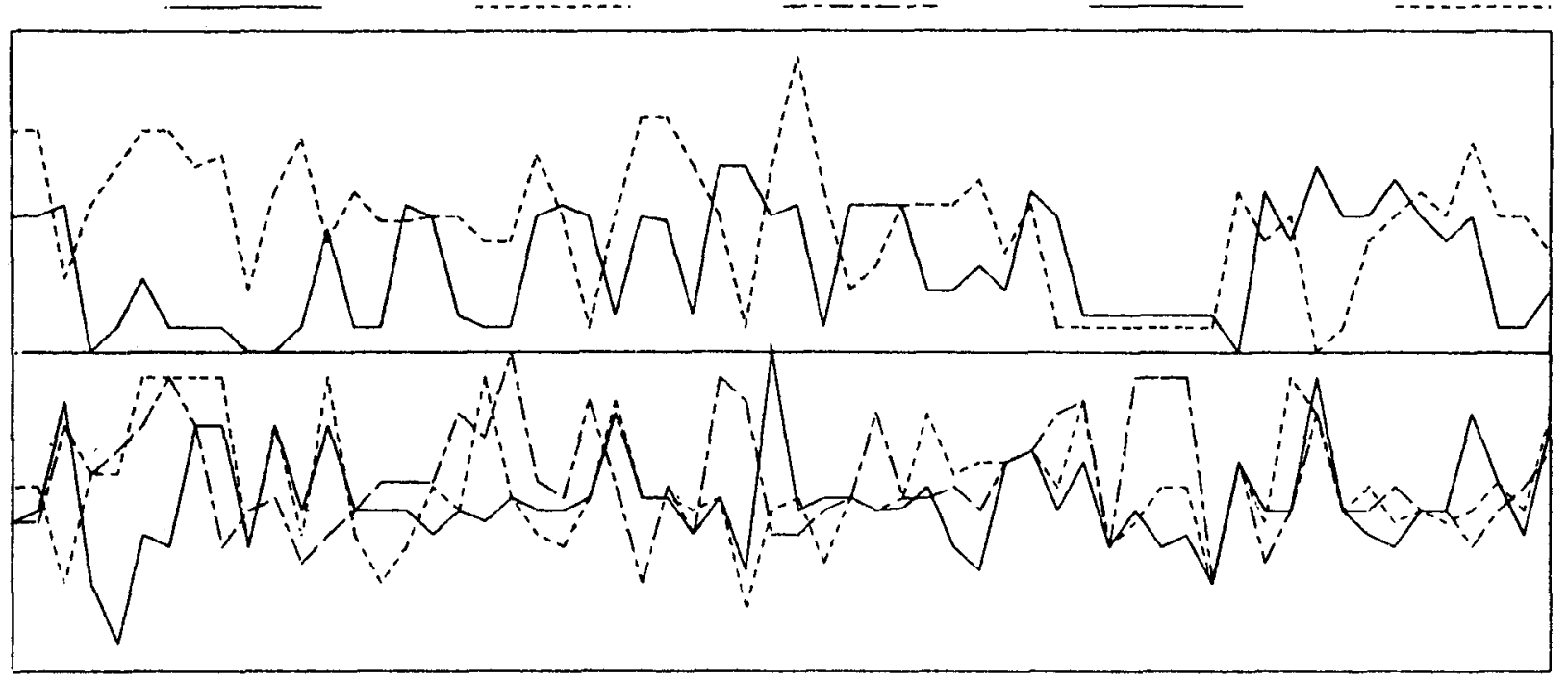

DISTANCES RELATIVE TO ANN

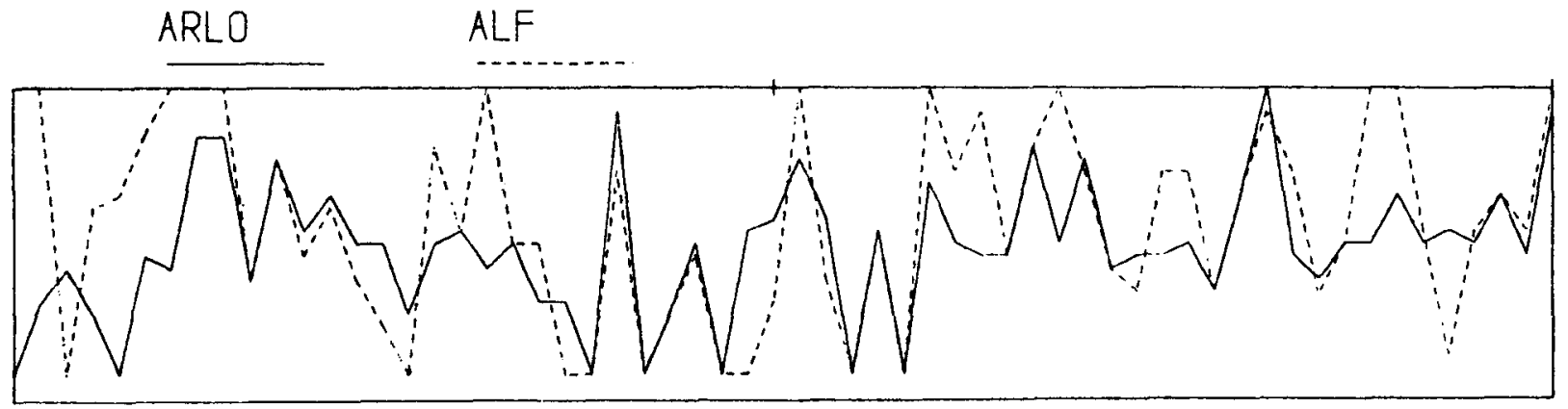

Fig. 2

of the routine is a loop over the number of separate plots required, one plot for each of the groups referred to in Section II. These plots are first annotated with the name of the focal member of the group, and, since there is usually a number of members in each group, a different line type (full, dotted, dashed, etc.) is associated with each member, this coding is written out with the annotation (Fig. 2).

The size of each plot is specified by input parameters, and a box is now drawn around the plot area. However, if there is more than a certain number of members in the group, the plot would be difficult to decipher with many lines occurring on top of each other. To make the plots more legibile, the $\mathrm{Y}$ dimension (separation) of the plot is doubled, and the lines for each half of the group are plotted either side of the zero-separation base line. Finally, the lines for each member can be drawn. To minimize pen-movement time, these lines are drawn in alternating directions, the data for the first $S$ is processed in the order of increasing time and the line is drawn from left to right, while for the next member the data is processed in decreasing time order and the corresponding line is drawn from right to left. Before returning to the calling routine, GHOP resets the variables specifying the plotter space in case further data is to be processed.

\section{(6) Subroutine HISTOP(INDEX)}

Called from: CHIMP, HPRINT. Calls: COPY8, COMP8.

This routine is responsible for producing the location occupation histograms. The data for these histograms is extracted from the array IHIST, where is has been stored by CHIMP, and the argument indicates to HISTOP which section of IHIST is to be processed. The routine assigns only 25 lines to each histogram, so that first a vertical scale factor must be calculated. This scale factor will be applied to the histograms for all Ss. Assigning 25 
THERE ARE SIX SUBJECTS, NAMED

PERCY ARLO ANN ALF BETTY BEBE

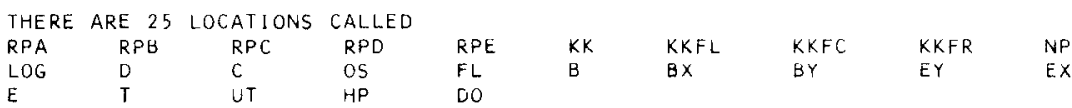

THE INTERLOCATION DISTANCES ARE

$\begin{array}{lllllllllllllllllllllll}0.00 & 0.31 & 0.61 & 0.91 & 1.22 & 0.61 & 0.76 & 0.91 & 1.37 & 0.91 & 1.07 & 2.29 & 3.20 & 3.66 & 2.44 & 2.03 & 3.05 & 2.90 & 2.74 & 2.59\end{array}$

$\begin{array}{llllllllllllllllllllll}2.59 & 2.44 & 2.44 & 2.64 & 1.98 & 0.31 & 0.00 & 0.31 & 0.62 & 0.91 & 0.46 & 0.91 & 0.92 & 1.22 & 0.91 & 1.07 & 2.29 & 3.20 & 3.66 & 2.29\end{array}$

$\begin{array}{lllllllllllllllllllll}3.05 & 2.90 & 2.74 & 2.64 & 2.44 & 2.44 & 2.29 & 2.29 & 2.44 & 1.68 & 0.61 & 0.31 & 0.00 & 0.31 & 0.62 & 0.31 & 1.07 & 0.76 & 0.91 & 0.92\end{array}$

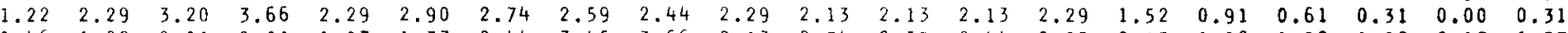

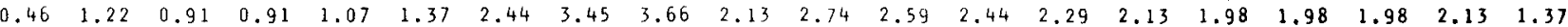

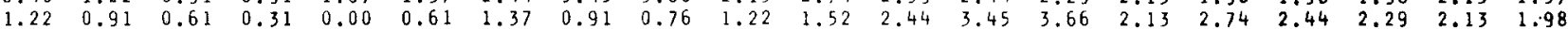

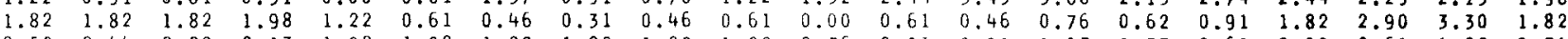

$\begin{array}{lllllllllllllllllllllll}2.59 & 2.44 & 2.29 & 2.13 & 1.98 & 1.98 & 1.82 & 1.82 & 1.82 & 1.22 & 0.76 & 0.91 & 0.91 & 1.07 & 1.37 & 0.61 & 0.00 & 0.61 & 1.22 & 0.31\end{array}$

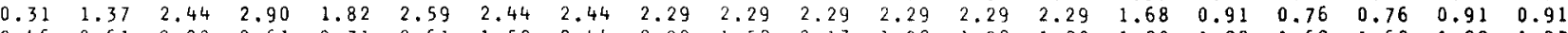

$\begin{array}{lllllllllllllllllllll}0.46 & 0.61 & 0.00 & 0.61 & 0.31 & 0.61 & 1.52 & 2.44 & 2.90 & 1.52 & 2.13 & 1.98 & 1.98 & 1.82 & 1.82 & 1.82 & 1.68 & 1.68 & 1.82 & 1.07\end{array}$

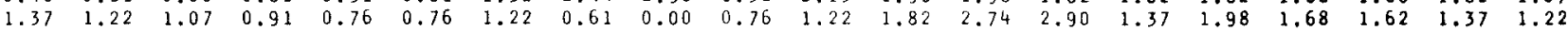

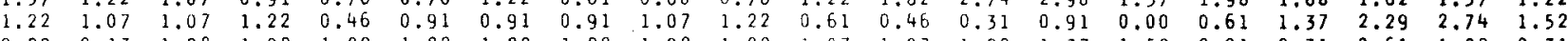

$\begin{array}{lllllllllllllllllllllll}2.29 & 2.13 & 1.98 & 1.98 & 1.82 & 1.82 & 1.82 & 1.82 & 1.98 & 1.22 & 1.07 & 1.07 & 1.22 & 1.37 & 1.52 & 0.91 & 0.31 & 0.61 & 1.22 & 0.31\end{array}$

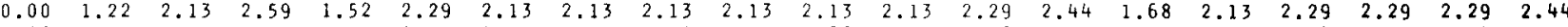

$\begin{array}{llllllllllllllllllll}1.82 & 1.37 & 1.52 & 1.82 & 1.37 & 1.07 & 0.00 & 0.91 & 1.52 & 1.07 & 1.68 & 1.82 & 1.98 & 1.98 & 2.13 & 2.29 & 2.44 & 2.59 & 2.74 & 2.13\end{array}$

$\begin{array}{lllllllllllllllllllll}3.30 & 3.30 & 3.45 & 3.45 & 3.45 & 2.90 & 2.44 & 2.44 & 2.74 & 2.29 & 2.13 & 0.91 & 0.00 & 0.76 & 1.52 & 1.82 & 1.95 & 2.13 & 2.44 & 2.59\end{array}$

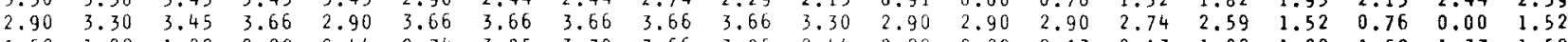

$\begin{array}{llllllllllllllllllll}1.52 & 1.82 & 1.82 & 2.29 & 2.44 & 2.74 & 3.05 & 3.30 & 3.66 & 3.05 & 2.44 & 2.29 & 2.29 & 2.13 & 2.13 & 1.82 & 1.82 & 1.52 & 1.37 & 1.52\end{array}$

$\begin{array}{llllllllllllllllllll}1.52 & 1.07 & 1.52 & 1.52 & 0.00 & 0.76 & 0.76 & 0.76 & 0.91 & 1.07 & 1.37 & 1.68 & 1.82 & 1.98 & 1.52 & 2.03 & 3.05 & 2.90 & 2.74 & 2.74\end{array}$

$\begin{array}{llllllllllllllllllll}2.59 & 2.59 & 2.13 & 1.98 & 2.29 & 2.29 & 1.68 & 1.82 & 1.52 & 0.76 & 0.00 & 0.31 & 0.61 & 0.76 & 1.07 & 1.37 & 1.82 & 1.98 & 2.13 & 1.82\end{array}$

$\begin{array}{lllllllllllllllllllll}3.05 & 2.90 & 2.74 & 2.59 & 2.44 & 2.44 & 2.44 & 1.98 & 1.68 & 2.13 & 2.13 & 1.82 & 1.95 & 1.82 & 0.76 & 0.31 & 0.00 & 0.31 & 0.46 & 0.76\end{array}$

$\begin{array}{llllllllllllllllllll}1.07 & 1.52 & 1.68 & 1.82 & 1.52 & 2.90 & 2.74 & 2.59 & 2.44 & 2.29 & 2.29 & 2.44 & 1.98 & 1.62 & 1.98 & 2.13 & 1.98 & 2.13 & 1.82 & 0.76\end{array}$

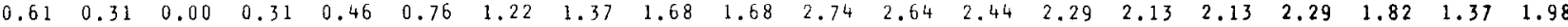

$\begin{array}{llllllllllllllllllll}2.13 & 1.98 & 2.44 & 2.29 & 0.91 & 0.76 & 0.46 & 0.31 & 0.00 & 0.31 & 0.46 & 0.91 & 1.07 & 1.37 & 1.22 & 2.59 & 2.44 & 2.29 & 2.13 & 1.98\end{array}$

$\begin{array}{llllllllllllllllllllll}1.98 & 2.29 & 1.82 & 1.22 & 1.82 & 2.13 & 2.13 & 2.59 & 2.44 & 1.07 & 1.07 & 0.76 & 0.46 & 0.31 & 0.00 & 0.31 & 0.76 & 0.91 & 1.07 & 1.07\end{array}$

$\begin{array}{lllllllllllllllllllll}2.59 & 2.44 & 2.13 & 1.98 & 1.82 & 1.98 & 2.29 & 1.82 & 1.22 & 1.82 & 2.13 & 2.29 & 2.90 & 2.74 & 1.37 & 1.37 & 1.07 & 0.76 & 0.46 & 0.31\end{array}$

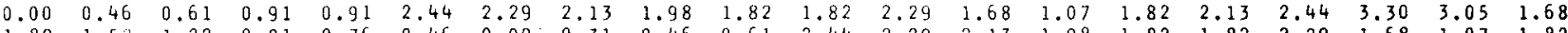

$\begin{array}{llllllllllllllllllll}1.82 & 1.52 & 1.22 & 0.91 & 0.76 & 0.46 & 0.00 & 0.31 & 0.46 & 0.61 & 2.44 & 2.29 & 2.13 & 1.98 & 1.82 & 1.82 & 2.29 & 1.68 & 1.07 & 1.82\end{array}$

$\begin{array}{lllllllllllllllllllll}2.29 & 2.59 & 3.45 & 3.30 & 1.82 & 1.98 & 1.68 & 1.37 & 1.07 & 0.91 & 0.61 & 0.31 & 0.00 & 0.31 & 0.61 & 2.64 & 2.44 & 2.29 & 2.13 & 1.98\end{array}$

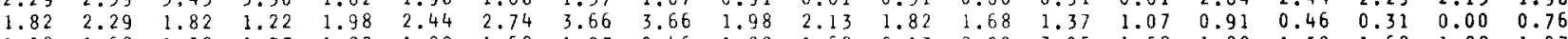

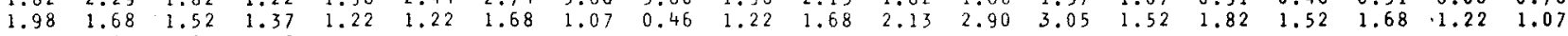

$0.91 \quad 0.610 .61 \quad 0.76 \quad 0.00$

HISTOGRAMS OF LOCATION POSITION TO BE PRINTED EVERY 500 MIN.

THE OBSERVATIONS ARE MADE EVERY 2 MIN,

AND THE MAXIMUM PERIOD OF OBSERVATION IS 500 MIN.

PLOTTER CHARACTERISTICS

$X S I Z E=20.00 \quad$ YSIZE $=4.00$

DISTANCES REQUIRED RELATIVE TO THREE CENTERS

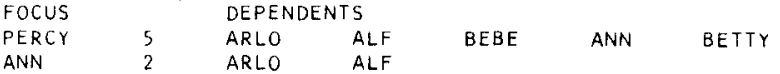

BETTY

BEBE

IITLE OF DATA IS DATA FROM 14 DECEMBER 1972

Fig. 3.

PERCH POSITIONS CTOTAL

SCALE FACTOR FOR HISTOGRAMS IS 2

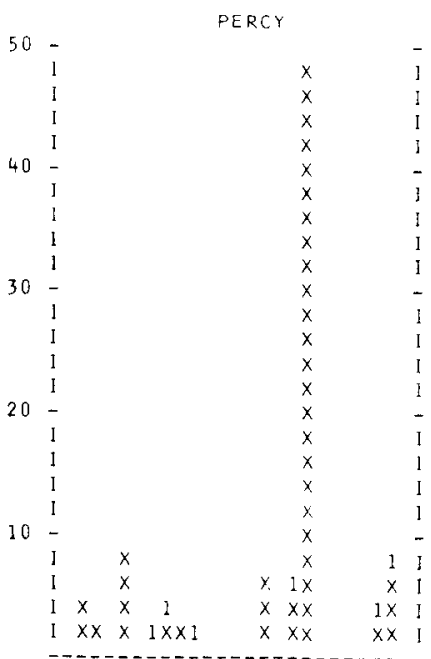

DATA FROM 14 DECEMBER 1972

ARLO

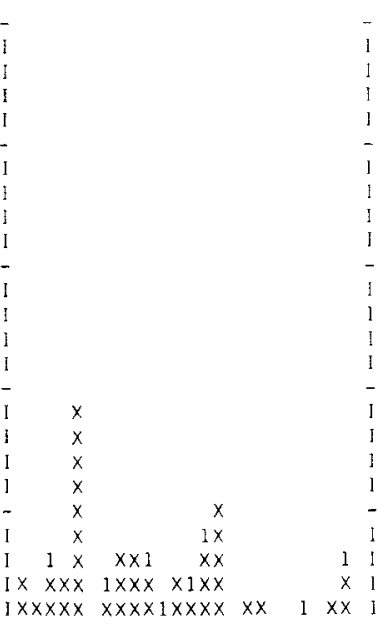

ANN

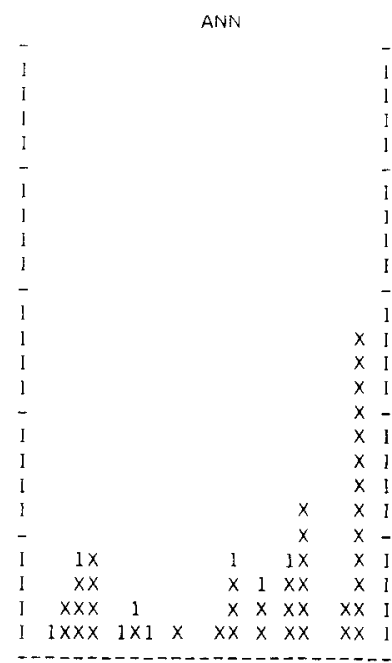

Fig. 4. 


$\begin{array}{lccccccc}\text { LOCATION } & \text { TOTAL } & \text { PEROY } & \text { ARLO } & \text { ANN } & \text { ALF } & \text { BET TY } & \text { BEBE } \\ \text { RPA } & 4 & 0 & 5 & 2 & 7 & 3 & 8 \\ \text { RPB } & 2 & 3 & 2 & 2 & 0 & 3 & 3 \\ \text { RPC } & 3 & 2 & 6 & 3 & 7 & 1 & 1 \\ \text { RPD } & 4 & 0 & 5 & 6 & 8 & 2 & 2 \\ \text { RPE } & 9 & 7 & 17 & 8 & 8 & 4 & 8 \\ \text { KK } & 0 & 0 & 0 & 0 & 0 & 0 & 1 \\ \text { KKFL } & 2 & 1 & 3 & 1 & 3 & 2 & 2 \\ \text { KKFC } & 5 & 3 & 6 & 3 & 8 & 4 & 4 \\ \text { KKFR } & 5 & 3 & 7 & 3 & 5 & 2 & 11 \\ \text { NP } & 3 & 1 & 7 & 0 & 8 & 1 & 3 \\ \text { LOG } & 1 & 0 & 1 & 2 & 3 & 0 & 1 \\ \text { D } & 2 & 0 & 6 & 0 & 3 & 1 & 3 \\ \text { C } & 1 & 0 & 3 & 0 & 4 & 0 & 2 \\ \text { OS } & 4 & 0 & 6 & 3 & 5 & 8 & 3 \\ \text { FL } & 10 & 6 & 15 & 6 & 13 & 8 & 13 \\ \text { B } & 1 & 0 & 0 & 0 & 2 & 0 & 3 \\ \text { BX } & 4 & 7 & 2 & 5 & 1 & 7 & 5 \\ \text { BY } & 12 & 58 & 3 & 0 & 0 & 9 & 3 \\ \text { EY } & 3 & 0 & 0 & 7 & 1 & 10 & 0 \\ \text { EX } & 4 & 0 & 0 & 13 & 1 & 6 & 3 \\ \text { E } & 1 & 0 & 1 & 0 & 0 & 1 & 3 \\ \text { T } & 1 & 0 & 0 & 0 & 2 & 0 & 2 \\ \text { UT } & 3 & 3 & 3 & 3 & 2 & 6 & 3 \\ \text { HP } & 16 & 8 & 5 & 35 & 8 & 24 & 15 \\ \text { DO } & 0 & 0 & 0 & 0 & 3 & 0 & 0\end{array}$

Fig. 5. Percentage of time spent in each location. next. In our study of six Ss and 25 locations, HISTOP produced two rows of three histograms with 14 spaces between each histogram.

To produce a number of histograms per row, the routine sets up a print buffer for each line. First, the vertical axes characters and the interhistogram space characters are inserted in the buffer. Then, for each row, the routine loops over the 25 lines assigned to the histograms. Each line corresponds to some value on the vertical axis, or some range of values if the scale factor is greater than 1 . This value is compared with the occupation count for each location and for all data to be processed in the row. If the value and count correspond, an " $X$ " is inserted in the print buffer. If the scale factor is greater than 1 , other characters are used as well. When all the data for a line have been examined, the buffer is printed out and the next line is processed. The print buffer is not reset between lines, because once any character position has been " $\mathrm{X}$ "-filled, this character will
THE MEAN DISTANCE
THE METWEEN PERCY
THE MEAN DISTANCE
BETWEEN PERCY
THE MEAS DISTANCE BETWEEN PERCY
THE MEAN DISTANCE BETWEEN PERCY
THE MEAN DISTANCE BETWEEN PERCY
THE MEAN DISTANCE BETWEEN ARLO
THE MEAN DISTANCE BETWEN ARLO
THE MEAN DISTANCE BETWEEN ARLO
THE MEAN DISTANCE BETWEEN ANN
THE MEAN DISTANCE BETWEEN ANN
THE MEAN DISTANCE BETWEEN ANN
THE MEAN DISTANCE BETWEEN ALF
THE MEAN DISTANCE BETWEEN ALF
THE MEAN DISTANCE BETWEEN BETTY

AND ARLO ANO ANN AND ALF AND BETTY AND BEBE AND ANN AND ALF AND BETTY AND BEBE AND ALF AND BETTY AND BEBE AND BETTY AND BEBE AND BEBE

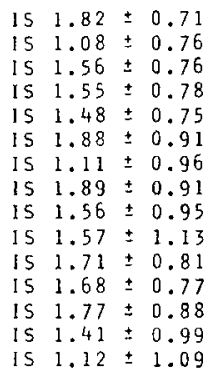

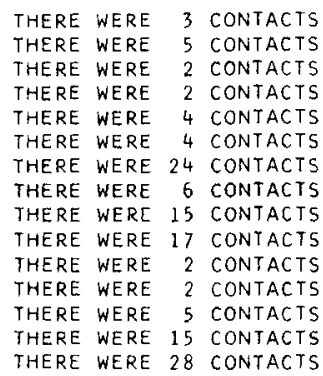

THERE WERE 28 CONTACTS

Fig. 6.

always be required. Finally, for the row, the buffer is used to print out the horizontal axis characters.

\section{(7) Subroutine HPRINT}

Called from: CHIMP. Calls: HISTOP.

As mentioned in Section II, the program has facilities for printing the histograms at the end of each user-defined interval, in addition to the final histograms which are produced at the end of the observation period. The data for these histograms are stored in the three-dimensional array, IHIST, where the third index, either 1 or 2 , indicates the interval and total histograms, respectively. In Subroutine CHIMP, data are added into the storage area for the interval histogram only. When the end of an interval is reached, CHIMP calls HPRINT, which immediately calls HISTOP, with Argument 1, to print out the interval histograms. On return from HISTOP, the data accumulated in the interval area of IHIST is added into the total histogram area, and the interval area is zeroed prior to the retum to CHIMP.

\section{(8) Subroutine PER1 $9 \emptyset$}

Called from: CHIMP.

This is the routine in which the summary table of the location histograms is produced. Apart from setting up the factors for conversion from absolute to percentage histograms per row is determined by the number of locations in the environment, and this number and the number of spaces between each histogram are calculated lines implies that two rows of histograms can be produced per page of output; however, the number of 
occupation, the routine consists of two nested loops. The outer loop is over the number of locations and the inner over the number of Ss. Within these loops, the occupation of each location by each $\mathrm{S}$ is extracted from the array IHIST and converted to a percentage of the total number of observations. The occupation of each location by all the Ss is totaled from the individual data, and both sets of information are printed.

\section{MACHINE-DEPENDENT ROUTINES}

As mentioned in the introduction, the program as supplied is entirely written in I.S.O. Standard FORTRAN; however, this program is incomplete. The majority of the omissions are connected to the specific structure of the Graphical Output Package, but there are two additional routines required for the comparison and assignment of alphanumeric data. On the I.C.L. 1906A, where the program is now used, the FORTRAN system does not include the handling of alphanumerics, and so the program calls on two library routines, COMP8 and COPY8, to compare and assign character data. The equivalent of these routines for any other machine must be provided, and a full specification is included in the program listing.

The Graphical Output Package used is a subset of the GHOST system (University of Birmingham, 1973) and routines similar to the 10 referenced by the program are probably available in most systems. Again, the routine specifications are included with the program. These routines handle the definition of the plotter area (FRAME, REGION, LIMITS, and GREND), the writing of character strings (CRSIZE and PLOTCL), and the drawing of lines in either full or hatched mode (POINT, JOIN, FULL, and BROKEN).

\section{NOTES ON USING THE PROGRAM}

In order to indicate the operation of the program,
Figs. 2-7 are samples of the output information, the data for this test run being provided with the program. Figure 2 shows the graph plotter output. Here the separation is plotted along the vertical axis and time along the horizontal axis, and two groups of Ss have been considered. The lower plot, for two dependent members, is the straightforward plot, whereas in the upper plot, for five members, the group has been divided into two subgroups and the lines for the two subgroups have been drawn either side of the central zero-separation base line. The other figures (3-7) are of the output produced on the line printer. Figure 3 is the summary listing of the fixed input data, while Figs. 4, 5, 6 , and 7 show, respectively, the final histograms of location, the summary table obtained from these histograms, the mean distances between all pairs of Ss, and the tables of the relative position frequency for all pairs.

The program as supplied allowed for a maximum of $10 \mathrm{Ss}, 40$ locations, and 500 observations in one period, although the latter limitation is overcome in a crude fashion since summary information is produced after the 500th observation and the storage locations are zeroed. However, as indicated in Section II, the arrays which set these limits are grouped in the COMMON statements according to these three parameters, and it is a simple task to alter the array dimensions. Further, the program uses three parameters, IDIMS, IDIMP, IDIMO, set up in a DATA statement in Subroutine CHIMP, which test that the supplied data does not exceed the dimensions of the storage arrays.

\section{REFERENCE}

University of Birmingham Computer Centre, Users Manual, Section 10: Graphics, 1973.

(Received for publication April 19, 1974; accepted May 1, 1974.) 